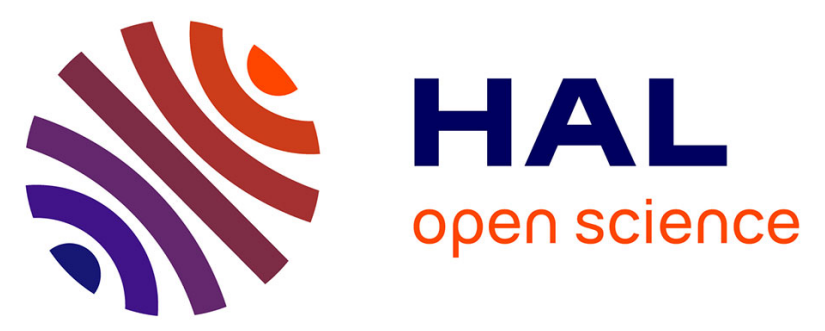

\title{
HARP-I: A Harmonic Phase Interpolation Method for the Estimation of Motion from Tagged MR Images
}

\author{
Hernán Mella, Joaquín Mura, Hui Wang, Michael D Taylor, Radomir
}

Chabiniok, Jaroslav Tintera, Julio Sotelo, Sergio Uribe

\section{- To cite this version:}

Hernán Mella, Joaquín Mura, Hui Wang, Michael D Taylor, Radomir Chabiniok, et al.. HARP-I: A Harmonic Phase Interpolation Method for the Estimation of Motion from Tagged MR Images. IEEE Transactions on Medical Imaging, 2021, 40 (4), pp.1240-1252. hal-03112239

\section{HAL Id: hal-03112239 \\ https://hal.inria.fr/hal-03112239}

Submitted on 16 Jan 2021

HAL is a multi-disciplinary open access archive for the deposit and dissemination of scientific research documents, whether they are published or not. The documents may come from teaching and research institutions in France or abroad, or from public or private research centers.
L'archive ouverte pluridisciplinaire HAL, est destinée au dépôt et à la diffusion de documents scientifiques de niveau recherche, publiés ou non, émanant des établissements d'enseignement et de recherche français ou étrangers, des laboratoires publics ou privés. 


\title{
HARP-I: A Harmonic Phase Interpolation Method for the Estimation of Motion from Tagged MR Images
}

\author{
Hernán Mella, Joaquín Mura, Hui Wang, Michael D. Taylor, Radomir Chabiniok, Jaroslav Tintera, Julio \\ Sotelo, and Sergio Uribe
}

\begin{abstract}
We proposed a novel method called HARP-I, which enhances the estimation of motion from tagged Magnetic Resonance Imaging (MRI). The harmonic phase of the images is unwrapped and treated as noisy measurements of reference coordinates on a deformed domain, obtaining motion with high accuracy using Radial Basis Functions interpolations. Results were compared against Shortest Path HARP Refinement (SP-HR) and Sine-wave Modeling (SinMod), two harmonic image-based techniques for motion estimation from tagged images. HARP-I showed a favorable similarity with both methods under noise-free conditions, whereas a more robust performance was found in the presence of noise. Cardiac strain was better estimated using HARP-I at almost any motion level, giving strain maps with less artifacts. Additionally, HARP-I showed better temporal consistency as a new method was developed to fix phase jumps between frames. In conclusion, HARP-I showed to be a robust method for the estimation of motion and strain under ideal and non-ideal conditions.
\end{abstract}

\section{Index Terms}

Cardiac MRI, Tagged MRI, SPAMM, CSPAMM, Harmonic Phase Analysis, Sine-wave Modeling, Cardiac Strain, Motion Estimation.

\section{INTRODUCTION}

C ARDIAC diseases are one of the leading causes of death globally (World Health Organization, 2018). Most of these diseases induce changes in the functionality of the cardiac muscle, leading to abnormal ventricular contractions [1]-[3]. The assessment of the global cardiac morphology and function has been extensively used for the diagnosis of cardiopathies. However, these metrics can suffer from preload and afterload dependency, geometric assumptions, moderate reproducibility, and its prediction capabilities are affected by conditions such as diabetes and hypertension [4], or congenital heart diseases [5]. Cardiac strain, on the other hand, is an advanced measure of the cardiac function with good reproducibility [6], which has demonstrated to be a powerful tool for the assessment and diagnosis of several cardiac diseases, such as heart failure, cardiomyopathies, dyssynchrony, abnormal pressures, and valve lesions [7]. Moreover, cardiac strain has the potential to detect subtle decreases in contractility not seen by ejection fraction. Therefore, accurate time resolved maps of the motion and deformation of the heart walls are needed for a better diagnosis and understanding of these diseases.

Currently, echocardiography is the most widely used technique to study areas in the left-ventricular walls with reduced functionality by estimating cardiac strain. [7], [8]. However, this technique suffers from low Signal-to-Noise Ratio (SNR), poor acoustic windows [9], geometric assumptions in the bidimensional case [10], and high inter-observer variability depending

The authors thankfully acknowledge the financial support of the ANID Ph. D. Scholarship 21170592 as well as the following projects and institutions: ANID-FONDECYT 1181057, ANID-FONDECYT Postdoctorado 3170737, ANID-FONDECYT de Iniciación en Investigación 11200481, ANID-Millennium Science Initiative Program - NCN17_129; the Ministry of Health of the Czech Republic (project No. NV19-08-00071), and InriaUTSW Medical Center Dallas Associated Team TOFMOD.

$\mathrm{H}$. Mella is with for the Biomedical Imaging Center and Department of Electrical Engineering, Pontificia Universidad Católica de Chile, Santiago, Chile (e-mail: hmella@uc.cl).

J. Mura is with for the Department of Mechanical Engineering, Universidad Técnica Federico Santa María, Santiago, Chile (e-mail: joaquin.mura@usm.cl).

H. Wang is with Philips, Cincinnati, USA, and the Department of Radiology, Cincinnati Children's Hospital Medical Center, Cincinnati, USA (e-mail: hui.wang1@philips.com).

MD. Taylor is with the Heart Institute, Department of Pediatrics, Cincinnati Children's Hospital Medical Center, Cincinnati, USA (e-mail: michael.taylor1@cchmc.org).

R. Chabiniok is with Inria, France; LMS, Ecole Polytechnique, CNRS, Institut Polytechnique de Paris, France; the Department of Mathematics, Faculty of Nuclear Sciences and Physical Engineering, Czech Technical University, Prague, Czech Republic; and the Division of Pediatric Cardiology, Department of Pediatrics, UT Southwestern Medical Center, Dallas, USA (e-mail: radomir.chabiniok@inria.fr).

J. Tintera is with the Institute for Clinical and Experimental Medicine, Prague, Czech Republic (e-mail: jaroslav.tintera@ikem.cz).

J. Sotelo is with the School of Biomedical Engineering, Universidad de Valparaíso, Valparaíso, Chile (e-mail: julio.sotelo@uv.cl).

S. Uribe is with the Biomedical Imaging Center, Department of Electrical Engineering, and Department of Radiology, Pontificia Universidad Católica de Chile, Santiago, Chile (e-mail: suribe@uc.cl).

H. Mella, J. Mura, J. Sotelo, and S. Uribe are with the Millennium Nucleus for Cardiovascular Magnetic Resonance, ANID - Millennium Science Initiative Program, Santiago, Chile. 
on the image quality [11]. Tagged Magnetic Resonance Imaging (MRI) has also been used for the assessment of the cardiac function [12]. In this technique, a grid pattern generated over the tissue at the beginning of the cardiac cycle (by a selective saturation of the tissue in the form of lines) follows the cardiac motion, allowing the tracking of the cardiac walls with temporal resolutions in the range of 30 to $50 \mathrm{msec}$.

At the beginning of the cardiac cycle, tag-lines in tagged images are parallel and equispaced. In the spatial frequency domain, this behavior is expressed as several spectral peaks with harmonic frequencies, which become wider due to the tissue contraction and energyless as the magnetization relaxes. Several techniques have been developed to acquire tagged MR images. One of the earlier approaches combined a series of selective RF pulses to create saturation bands on the cardiac tissue [13]. A year later, the Spatial Modulation of Magnetization (SPAMM) was introduced [14], which employed a position encoding gradient and two non-selective RF pulses to rapidly saturate the magnetization. A significant improvement was made with the Complementary SPAMM (CSPAMM) sequence [15], which using two SPAMM acquisitions, removed the non-tagged signal from the image at the cost of doubling the acquisition time. Additionally, other techniques have been introduced, such as high-order SPAMM, slice-following CSPAMM, DANTE, and variations of both of them [16], [17].

Lately, new acquisition strategies were developed to acquire 3D tagging data. In [18] a 3D CSPAMM sequence was proposed to estimate 3D cardiac motion, taking 16 minutes to acquire all the information. A further improvement was made in [19], where an accelerated 3D CSPAMM was introduced, allowing the acquisition of the whole heart in only 3 breath-holds of 18 heart-beats duration each.

With the advances in the imaging sequences, many motion estimation strategies based on the image intensity or its kspace were developed. In the first group, tag-lines tracking, optical flow, and image registration methods, which use intensity models and brightness constraints, are found [20]-[23]. Additionally, although computationally expensive, the image registration problem has been enriched with biomechanical models of the heart to improve the tracking outcome [24], [25]. The other group of techniques are those based on the identification and filtering of the harmonic spectral peaks, such as the Harmonic Phase (HARP) analysis [26], [27], Sine-Wave Modeling (SinMod) [28] technique, and Gabor filter banks [29], [30]. The three methods use bandpass filters to obtain images containing motion information processed in three different ways: HARP estimates motion using local approximations of the phase gradient, SinMod does it using power spectrums, and Gabor filter banks detecting tag-lines changes through several k-space filtered images [31]. However, until today, the most used techniques are HARP analysis and SinMod method.

Since its initial development, several approaches have been proposed to improve the capabilities of HARP, including the Improved HARP [32], Extended HARP [33], dense multiscale HARP [34], seeded region growing refinement [35], shortest path HARP refinement [36] (SP-HR), and HARP tracking with locally uniform deformation assumption [37], which using spatial and/or temporal constraints, were developed to fix tracking issues due to large deformations between image frames, throughplane motion, and tissue boundaries. For SinMod, few improvements have been proposed (at least for the author knowledge), being worth to mention the robust and accurate center-frequency estimation (RACE) algorithm [38] and the multilevel B-splines based method [39]. Additionally, HARP and SinMod have been successfully extended to work with 3D data [18], [40], [41].

Although HARP-based techniques and SinMod are good methods for estimating motion and strain, both techniques are affected by different issues: HARP could be sensitive to the image quality [28], SinMod over-estimates strain and angular motions [42], and both can be very sensitive to DC contamination, as will be shown later. To tackle the issues described above, a robust and fast Harmonic Phase Interpolation method (HARP-I) is introduced. Similar to HARP and SinMod, our proposed method operates using bandpass filters to obtain the harmonic phase of the image. Then, unlike HARP-based methods and SinMod, the whole phase is corrected from wrapping artifacts and temporal inconsistencies, which leads to a function that moves accordingly with the heart motion. Finally, the displacement field on a reference domain is obtained using a Radial Basis Functions (RBF) interpolation scheme defined on unwrapped phases.

In this study, we introduce HARP-I and compare its performance against SP-HR and SinMod using numerical phantoms and tagged MR images from 24 volunteers. We also present a sensitivity assessment under ideal (noise-free) and non-ideal (noisy) image conditions.

\section{Methods}

\section{A. Principle of HARP-I}

The HARP-I method is based on the same principle as SP-HR but using a different approach to estimate motion. Assuming that the harmonic phase of tagged images (corrected from wrapping artifacts) moves accordingly with the tissue [26], [27], the unwrapped phase of images tagged in orthogonal directions are used to define a new virtual coordinate system. Then using an interpolation scheme, motion maps are obtained on a fixed reference system.

The motion of a continuum body $\boldsymbol{R}_{0}$ is described using the deformation map $\boldsymbol{\varphi}$, which maps the coordinates $\boldsymbol{X} \in \boldsymbol{R}_{0}$ onto its position $\boldsymbol{x} \in \boldsymbol{R}_{k}$ (see Figure 1). Here $\boldsymbol{R}_{0}$ denotes the body at a reference frame (e.g., the heart at end-diastole) and $\boldsymbol{R}_{k}$ the region of the space occupied by the body at the time $t_{k}$ [43] (e.g., the beating heart). At this point should be noticed that the following description of the method is general and can be applied to either $2 \mathrm{D}$ and $3 \mathrm{D}$ data by repeating the whole procedure to an additional dimension. 


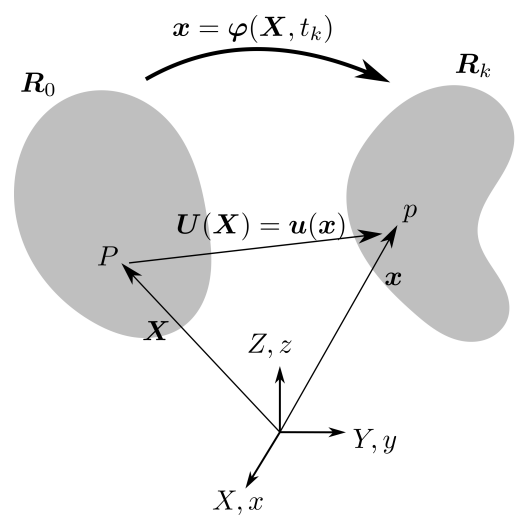

Fig. 1: Representation of motion of a continuum body. The motion of the body $\boldsymbol{R}_{0}$ is completely defined through the deformation map $\boldsymbol{\varphi}$, which maps every material point $\boldsymbol{P} \in \boldsymbol{R}_{0}$ to its position at a later state $\boldsymbol{p} \in \boldsymbol{R}_{k}$.
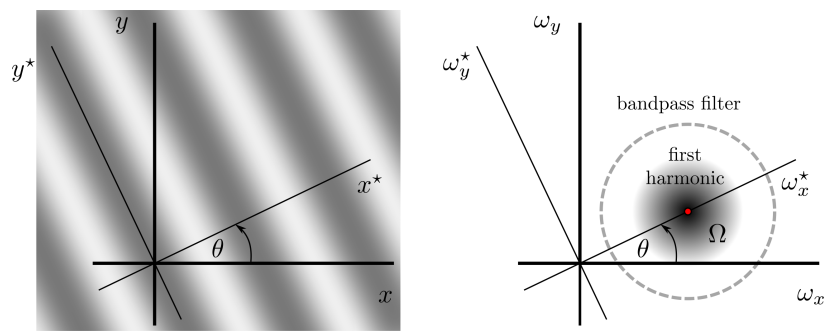

Fig. 2: (Left image) Tagged image with tag-lines pointing towards the oblique direction $x^{\star}$, given by the angulation $\theta$. (Right image) The Fourier transform of the tagging image gives a frequency spectrum in the domain $\left[\omega_{x}, \omega_{y}\right]$, with the first harmonic peak centered at a frequency $\Omega$ over the $\omega_{x}^{\star}$ axis. The central frequency of the bandpass filter (gray broken lines) coincides with $\Omega$.

Let us consider now the pair of images $I\left(\boldsymbol{x}, t_{0}\right)$ and $I\left(\boldsymbol{x}, t_{k}\right)$ of a sequence of tagged MR images not necessarily subsequent, in which brightness represents an object occupying the domains $\boldsymbol{R}_{0}$ and $\boldsymbol{R}_{k}$ at different times. Without loss of generality, the intensity distribution of the $k$-th image with tag-lines in the $X$ direction was modeled as

$$
I\left(\boldsymbol{x}, t_{k}\right)=A\left(t_{k}\right) \cos \left(\Omega X\left(\boldsymbol{x}, t_{k}\right)\right)+n\left(\boldsymbol{x}, t_{k}\right)
$$

where $\Omega$ represents the spatial frequency of the sinusoidal tag pattern, $A$ the image intensity depending on time $t_{k}$, and $n$ the noise contributions which depend separately of $\boldsymbol{x}$ and $t_{k}$ (i.e. vary with the position and time). Then, isolating the harmonic part of the image in (1) using a bandpass filter in the frequency domain $\left[\omega_{x}, \omega_{y}\right]$ with central frequency $\Omega$, we obtained the harmonic image

$$
I^{b f}\left(\boldsymbol{x}, t_{k}\right)=A\left(t_{k}\right) e^{i \Omega X\left(\boldsymbol{x}, t_{k}\right)}+n^{b f}\left(\boldsymbol{x}, t_{k}\right),
$$

where $n^{b f}$ is the bandpass filtered version of $n$. Taking the angle in (2), a wrapped version of the harmonic phase of $I$ was obtained:

$$
\phi_{w}\left(\boldsymbol{x}, t_{k}\right)=\angle I^{b f}\left(\boldsymbol{x}, t_{k}\right)=\mathcal{W}\left(\Omega X\left(\boldsymbol{x}, t_{k}\right)+n_{\phi}\left(\boldsymbol{x}, t_{k}\right)\right)
$$

where $\mathcal{W}(\phi)=\bmod (\phi+\pi, 2 \pi)-\pi$ denotes the non-linear wrapping operator and $n_{\phi}$ the phase noise induced by $n^{b f}$.

Following the same procedure for an image with tag-lines in the $Y$ direction, we can obtain the wrapped phase for the two directions $X$ and $Y$ as:

$$
\phi_{w}\left(\boldsymbol{x}, t_{k}\right)=\mathcal{W}\left(\Omega \boldsymbol{X}\left(\boldsymbol{x}, t_{k}\right)+\boldsymbol{n}_{\phi}\left(\boldsymbol{x}, t_{k}\right)\right)
$$

where $\boldsymbol{X}$ now denotes the two components of the reference system, and $\phi_{w}$ and $\boldsymbol{n}_{\phi}$ (in bold) the vectorial versions of the wrapped phases and noise components.

Differing from SP-HR, we corrected the wrapping artifacts in (4) to obtain a phase $\phi$ that depends directly on the Eulerian (spatial) description of the reference position $\boldsymbol{X}$, given by:

$$
\boldsymbol{\phi}\left(\boldsymbol{x}, t_{k}\right)=\Omega \boldsymbol{X}\left(\boldsymbol{x}, t_{k}\right)+\boldsymbol{n}_{\phi}\left(\boldsymbol{x}, t_{k}\right)+2 \pi \boldsymbol{c}_{k},
$$

where $2 \pi \boldsymbol{c}_{k}$ is a constant vector on $\boldsymbol{R}_{k}$ (with $\boldsymbol{c}_{k}$ a vector of integers) denoting the fact that phases obtained from the unwrapping procedure are not temporally consistent and can differ by a multiple of $2 \pi$. 

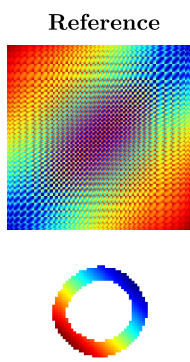

(a) phase 1
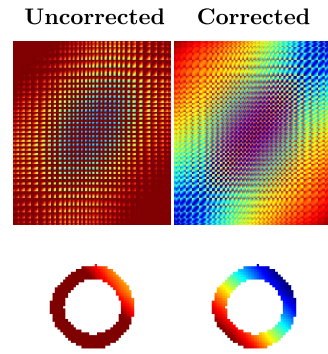

(b) Cardiac phase 5

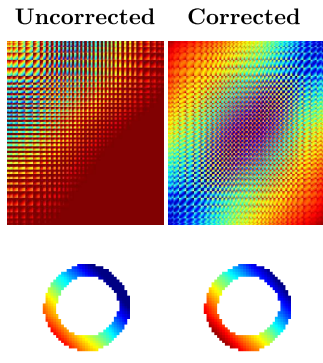

(c) Cardiac phase 12

Fig. 3: Phase-distance matrices (upper row) and the corresponding unwrapped harmonic phases (bottom row). Figure (a) shows the distance matrix and phase for the reference frame, whereas (b) and (c) show the uncorrected (left) and corrected (right) distance matrices and phases for frames 5 and 12. The matrices were obtained from an image of a healthy volunteer with taglines in the direction given by $\theta=45^{\circ}$ (see Figure 2) and using only the pixels inside the LV (this explains why the matrix size is square in (a) but non-square in (b) and (c). See (12) for the definition of distance matrices). The colors on all the plots share the same scale.

Using the first phase as reference (i.e., we assumed $c_{0}=0$ ), the reference coordinates are related with the new virtual system as:

$$
\boldsymbol{X}_{V}\left(\boldsymbol{x}, t_{k}\right)=\boldsymbol{\phi}\left(\boldsymbol{x}, t_{k}\right)-\boldsymbol{c}_{k}=\Omega \boldsymbol{X}\left(\boldsymbol{x}, t_{k}\right)+\boldsymbol{n}_{\phi}\left(\boldsymbol{x}, t_{k}\right)
$$

where $\boldsymbol{X}_{V}\left(\boldsymbol{x}, t_{k}\right)$ is a function that depends on noisy measurements of the reference position $\boldsymbol{X}\left(\boldsymbol{x}, t_{k}\right)$ on $\boldsymbol{R}_{k}$ (further details regarding the estimation of $\boldsymbol{c}_{k}$ are discussed later).

Using this new reference system, we defined a multivariate RBF interpolation scheme for noisy scattered data to map deformed coordinates into its reference description [44]. Thus, the interpolation scheme was defined as:

$$
\boldsymbol{g}(\boldsymbol{y})=\sum_{i=1}^{N_{\text {pixels }}} \lambda_{i} \cdot \psi\left(\left\|\boldsymbol{y}-\boldsymbol{X}_{V}\left(\boldsymbol{x}_{i}, t_{k}\right)\right\|\right)
$$

where $N_{\text {pixels }}$ denotes the number of pixels in the image, $\boldsymbol{g}(\boldsymbol{y})$ the interpolated function at the point $\boldsymbol{y}$ (with $\boldsymbol{y}$ a variable that can be evaluated at any point of the virtual system), $\psi\left(\left\|\boldsymbol{y}-\boldsymbol{X}_{V}\left(\boldsymbol{x}_{i}, t_{k}\right)\right\|\right)$ a radial basis function centered at $\boldsymbol{X}_{V}\left(\boldsymbol{x}_{i}, t_{k}\right)$, and $\lambda_{i}$ the weighting factor associated with the $i$-th basis function.

Instead of constructing the exact interpolation of polluted measurements (see Appendix A), we seek the approximation $\hat{\boldsymbol{g}}\left(\boldsymbol{X}_{V}\left(\boldsymbol{x}_{i}, t_{k}\right)\right) \approx \boldsymbol{x}_{i}$, using [45]:

$$
(\boldsymbol{\Psi}+\eta \mathbf{I}) \boldsymbol{\lambda}=\boldsymbol{G}
$$

where $\eta>0$ is a regularization constant that ensures the non-singularity of the system, I the identity matrix, $\boldsymbol{\lambda}$ a vector of weights, and $\boldsymbol{G}$ a vector of measurements (details about the matrix $\boldsymbol{\Psi}$ are in the Appendix VI-A).

After solving (8), the interpolator in (7) was evaluated at $\boldsymbol{X}_{V}\left(\boldsymbol{X}, t_{0}\right)$, allowing the estimation of the deformed position of the object $\boldsymbol{R}_{k}$ on the reference configuration $\boldsymbol{R}_{0}$ (see Appendix B). Finally. the displacement field $\boldsymbol{U}$ of the object $\boldsymbol{R}_{0}$ between the times $t_{0}$ and $t_{k}$ was easily estimated using

$$
\boldsymbol{U}\left(\boldsymbol{X}, t_{k}\right)=\varphi\left(\boldsymbol{X}, t_{k}\right)-\boldsymbol{X}
$$

\section{B. Strategies for correcting temporal phase inconsistencies}

For a proper estimation of displacements, $c_{k}$ is estimated using $\phi_{1}$ as reference phase.

Exploding the structure of the distance matrices: as we treat the harmonic phases as a material property, both of the following distance matrices should always have a similar structure:

$$
\begin{gathered}
r_{m n}^{\mathrm{ref}}=\left\|\boldsymbol{X}_{V}\left(\boldsymbol{X}_{m}, t_{0}\right)-\boldsymbol{X}_{V}\left(\boldsymbol{X}_{n}, t_{0}\right)\right\|, \\
r_{m n}^{k}=\left\|\boldsymbol{X}_{V}\left(\boldsymbol{X}_{m}, t_{0}\right)-\boldsymbol{X}_{V}\left(\boldsymbol{x}_{n}, t_{k}\right)\right\| .
\end{gathered}
$$

However, due to the phase jumps between temporal frames, the previous assertion is not valid (see Figure 3).

Phase inconsistencies are ligated to the constant $\boldsymbol{c}_{k}$, and can be fixed by ensuring the similitude between $\mathbf{r}^{\text {ref }}$ and $\mathbf{r}^{k}$. This condition is imposed through the coming optimization problem:

$$
\arg \min _{\boldsymbol{c}_{k}}\left\{\left|\min _{m, n} r_{m n}^{\mathrm{ref}}-\min _{m, n} r_{m n}^{k}\right|\right\},
$$


where $\boldsymbol{c}_{k}$ is defined implicitly on $\mathbf{r}^{k}$ through $\boldsymbol{X}_{V}\left(\boldsymbol{x}_{n}, t_{k}\right)$ (see (6)). This approach does not introduce any restriction on the temporal resolution and can be used on either segmented or non-segmented cardiac geometries.

\section{Application of HARP-I in oblique directions}

Although the whole process consider tag-lines in the directions $x$ and $y$, we can also apply HARP-I in oblique directions $x^{\star}$ and $y^{\star}$ (see Figure 2). Defining the angulation of the tag-lines as $\theta$, the deformed and frequency coordinates $\left[x^{\star}, y^{\star}\right]$ and $\left[\omega_{y}^{\star}, \omega_{y}^{\star}\right]$ (respectively) are defined by:

$$
\begin{aligned}
& {\left[\begin{array}{l}
x^{\star} \\
y^{\star}
\end{array}\right]=\left[\begin{array}{cc}
\cos \theta & \sin \theta \\
-\sin \theta & \cos \theta
\end{array}\right]\left[\begin{array}{l}
x \\
y
\end{array}\right],} \\
& {\left[\begin{array}{l}
\omega_{x}^{\star} \\
\omega_{y}^{\star}
\end{array}\right]=\left[\begin{array}{cc}
\cos \theta & \sin \theta \\
-\sin \theta & \cos \theta
\end{array}\right]\left[\begin{array}{l}
\omega_{x} \\
\omega_{y}
\end{array}\right] .}
\end{aligned}
$$

Thus, replacing $\boldsymbol{x}$ by $\boldsymbol{x}^{\star}=\left[x^{\star}, y^{\star}\right]$ and $\boldsymbol{\omega}$ by $\boldsymbol{\omega}^{\star}=\left[\omega_{x}^{\star}, \omega_{y}^{\star}\right]$ in (1) to (9) all the procedure remains valid.

\section{Implementation of HARP-I}

HARP-I method, listed in (1) to (9), was implemented in MATLAB (The MathWorks, Inc., Natick, Massachusetts). Similar to SP-HR and SinMod, Fast Fourier Transform (FFT) was applied to two input images (not necessarily subsequent) $I_{1}$ and $I_{k}$ with tag-lines in the direction $x^{\star}$ (or $y^{\star}$ ) (see Figure 2), leading to frequency spectrums in the $\left[\omega_{x}^{\star}, \omega_{y}^{\star}\right]$ domain. The center of the region containing the first harmonic component of the images was identified as $\left[\Omega_{x}, \Omega_{y}\right]$, and the central frequency $\Omega$ and the tag-lines angulation $\theta$ (see Figure 2) were calculated using:

$$
\Omega=\sqrt{\Omega_{x}^{2}+\Omega_{y}^{2}}, \quad \theta=\arccos \left(\frac{\Omega_{x}}{\Omega}\right)
$$

In practice, we identify just one harmonic component and choose the second direction to be perpendicular to the first one.

The harmonic phases given in (4) were corrected from wrapping artifacts using a quality-guided path following unwrapping algorithm [46], which has been previously used for the estimation of displacements from DENSE images [47], [48].

In our implementation we tested several families of RBF, such as Wendland's, Wu's, splines, Gaussians, and multiquadrics, being the last which had better smoothing properties without comprising the quality of the estimation. Therefore, we used a third-order multiquadric RBF given by

$$
\psi(r)=\left(r^{2}+a^{2}\right)^{3 / 2}, \quad a \in \mathbb{R}
$$

where $a$ is a constant that controls the smoothing during the interpolation process [49]. The value of $a$ used on all the experiments (with synthetic data and real images) was empirically determined as

$$
a=\frac{150}{\mathrm{~N}^{o} \text { pixels per wavelength }}=\frac{150 \Delta x}{2 \pi / \Omega},
$$

where $\Delta x$ denotes the pixel size. The key idea behind this choice is that as the number of pixels-per-wavelength decreases, higher frequency components are present in the filtered images and therefore, the amount of smoothing should increase.

\section{E. Implementation of SP-HR and SinMod}

To compare our method with other processing techniques, we used SP-HR and implemented SinMod methods as described in Osman et al. [26], [27], [36] and Arts et al. [28], respectively. In the case of SP-HR, the source code was provided by the Image Analysis and Communication Lab at Johns Hopkins University. For the SinMod method, the implementation was done in accordance with [28], [41]. The local quality model proposed by the authors, consisting on a weighted Hanning windowing of the power spectrum based on an intensity-based quality measure, was used to correct the motion estimation. The size of the Hanning window was set to 15 and the power of the quality measure was chosen as 8 .

Motion with SinMod was estimated in a frame-by-frame basis using two consecutive images, and tracked backward to a reference frame using a Thin-Plate spline interpolation. For the estimation of strain, no smoothing techniques were applied to the estimation of spatial derivatives. However, a 10th order polynomial was fitted to each component of the estimated motion to slightly smooth the tissue trajectories [48], [50], [51].

For the sake of fairness during the comparison, we used the same Butterworth bandpass filter [41] for the three methods, choosing the frequency-cutoff and decay order accordingly to ensure, as far as possible, the removal of higher harmonics and the DC component. The central frequency $\Omega$ of the filter was defined as the tag-lines frequency. 


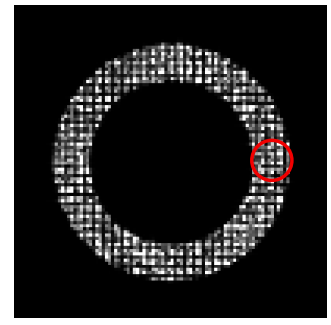

(a)

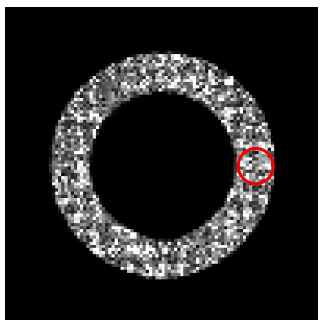

(b)

Fig. 4: (a) First and (b) last frames of a noisy synthetic data. The red circle denotes the place where the stiffer inclusion was added. The contrast of the images (see (18)) was set differently for a better visualization. Both images were obtained using the $\mathrm{WL}_{5}$

\section{EXPERIMENTS}

\section{A. In-silico experiments}

SP-HR, SinMod, and HARP-I were tested using a synthetic 2D dataset of 10 different idealized left-ventricular geometries. The intensity model used was similar to the given in (1) but with a decaying tagged and growing non-tagged terms as follows:

$$
I\left(\boldsymbol{x}, t_{k}\right)=1-e^{-t_{k} / T 1}+e^{-t_{k} / T 1} \cos \left(\Omega X\left(\boldsymbol{x}, t_{k}\right)\right),
$$

where $T 1=0.72$ denotes a relaxation parameter chosen to achieve a $75 \%$ of signal decay of the tagged part in the last frame. Each image was generated with a resolution of $200 \times 200$ pixels. The intensity model given in (18) emulates the signal of tagging MR images without DC correction [52].

The motion pattern of the idealized geometries combines angular and non-uniform radial displacements with a stiffer inclusion to simulate an abnormal contraction (see Figure 4). Motion maps were estimated under noise-free and noisy conditions, and all the experiments were performed using wavelengths of $2.9,4.9$, and 6.9 pixels, namely $\mathrm{WL}_{3}, \mathrm{WL}_{5}$, and $\mathrm{WL}_{7}$ respectively.

The maximum angular and radial displacements of the inner wall, $\Delta \theta$ and $\Delta r$ respectively, vary across data following:

$$
\Delta \theta=\frac{\mathrm{WL}_{i}}{2 r}, \quad \Delta r=\frac{\mathrm{WL}_{i}}{2}
$$

where $\mathrm{WL}_{i}$ is the wavelength of the intensity model $(i=4,5,7)$ and $r$ the inner radius. For the outer displacements, no angular motion was imposed and the maximum radial motion was estimated in order to ensure a isochoric deformation. The spatial variation of the motion pattern across the wall (between the inner and outer radius) was chosen to be linear, with a stiffer additive inclusion to simulate an abnormal contraction. The maximum displacement was applied in increments of $0.1 \mathrm{WL}_{i}$, i.e., six motion levels per data were simulated, being the maximum displacement achieved in the last frame.

To investigate the behavior of the three methods with low SNR images, additive uncorrelated white Gaussian noise with standard deviation 0.06 was added to both real and complex channels of all the images (see Figure 4).

\section{B. In-vivo experiments}

Finally, we tested and compared the three methods on a short-axis view of the left ventricle (different cardiac levels) of 23 volunteers (19 males of $28 \pm 15$ years and 4 females of $50 \pm 21$ years of which 9 were acquired on a 1.5T Ingenia system (Philips, Best, Netherlands), 4 on a 1.5T Achieva system (Philips, Best, Netherlands), and 10 on a 1.5T Avanto Fit system (Siemens Healthineers, Erlangen, Germany). The spacings of the tag-lines were $4.5 \pm 0.8$ pixels per wavelength.

Additionally, to demonstrate the functionality of HARP-I on 3D data, the method was tested on a 3D CSPAMM image of one healthy volunteer (male, 66 years old) acquired on a 1.5T Achieva system (Philips, Best, Netherlands). After alignment and resampling of the volumes in each direction, the size of the reconstructed matrix was $112 \times 112 \times 112$ with a voxel size of $0.96 \times 0.96 \times 1.12 \mathrm{~mm}^{3}$. The tag spacing was $7 \mathrm{~mm}$ in each direction. For further details about the acquisition, please refer to [19].

The acquisition of all the subjects was approved by the Institutional Review Boards at Pontificia Universidad Católica de Chile (Santiago, Chile), Cincinnati Children's Hospital Medical Center (Ohio, USA), and the Institute for Clinical and Experimental Medicine (Prague, Czech Republic). The dataset was composed of 16 healthy volunteers (including the 3D data) and 8 patients with known cardiopathies. In Table I, a summary of the diagnoses and/or MRI findings of the subjects can be found. 
TABLE I: Diagnoses and/or MRI findings for subjects with abnormal ventricular contraction

\begin{tabular}{|c|c|c|c|}
\hline Identifier & Sex & Age & Diagnosis/Specialist impression \\
\hline $2^{\star}$ & M & 21 & Mildly decreased LV ejection fraction (EF 50\%). \\
\hline $4^{\star}$ & $\mathrm{F}$ & 69 & $\begin{array}{l}\text { Severely decreased LV systolic function. Dysk- } \\
\text { inetic motion of apex (with thrombus present). } \\
\text { Possible hypertrophic cardiomyopathy in septum at } \\
\text { LV outflow tract. }\end{array}$ \\
\hline $7^{\star}$ & M & 43 & Globally hypokinetic LV. \\
\hline 10 & M & 12 & Duchenne muscular dystrophy. \\
\hline 11 & M & 30 & Duchenne muscular dystrophy. \\
\hline 12 & M & 11 & Duchenne muscular dystrophy. \\
\hline 13 & M & 12 & Hypertrophic cardiomyopathy. \\
\hline 14 & M & 13 & Duchenne muscular dystrophy. \\
\hline \multicolumn{4}{|c|}{$\begin{array}{l}\text { Each subject identifier is accompanied by its respective diagnosis and/or } \\
\text { MRI finding. Diagnoses were made by a cardiologist with expertise in } \\
\text { cardiovascular MRI, while MRI findings were made by a medical doctor } \\
\text { with more than } 5 \text { year experience with clinical cardiovascular MRI. These } \\
\text { identifiers will be used in the rest of the article to refer to the data. } \\
\text { ^: MRI finding; M: male; F: female; EF: ejection fraction. }\end{array}$} \\
\hline
\end{tabular}

\section{Statistical analysis}

All the results presented include comparisons between reference displacements and strain, considering displacement magnitude and direction, and circumferential and radial components of the Lagrangian strain tensor [43]. The metrics used to quantify the error were the normalized root Mean Square Error (nRMSE) and Directional Error (DE) [53], defined as:

$$
\begin{aligned}
\mathrm{nRMSE} & =\frac{1}{\max \left\|\boldsymbol{a}_{i}^{e}\right\|} \sqrt{\frac{1}{N} \sum_{i=1}^{N}\left\|\boldsymbol{a}_{i}-\boldsymbol{a}_{i}^{e}\right\|^{2}} \\
\mathrm{DE} & =\frac{180}{\pi N} \sum_{i=1}^{N} \arccos \left(\frac{\left|\boldsymbol{u}_{i} \cdot \boldsymbol{u}_{i}^{e}\right|}{\left\|\boldsymbol{u}_{i}\right\|_{2}\left\|\boldsymbol{u}_{i}^{e}\right\|_{2}}\right)
\end{aligned}
$$

where $\|\cdot\|$ represents the Euclidean norm, $N$ the number of analyzed pixels in the image, $\boldsymbol{a}_{i}$ a pixelwise scalar or vectorial quantity at pixel $i$, and $\boldsymbol{u}_{i}$ the pixelwise displacement. The superscript ()$^{e}$ denotes the reference value. All the results reported in the succeeding section are average values of the metrics presented in (20) and (21) computed over the whole synthetic dataset $(N=10)$ for each displacement level.

\section{REsults}

In the following sections, we split the results into three parts. The first and the second comprise the in-silico results of the noise-free and noisy experiments respectively, whereas the third one reports the results from the in-vivo data.

\section{A. Noise-free experiments}

Figure 5 shows the mean nRMSE and DE across the whole dataset calculated using the expressions given in (20) and (21). For all the wavelengths the three methods showed a similar behavior, achieving all of them the best performance at WL 3 and the worst at $\mathrm{WL}_{7}$ (see Figures 5a to 5c). Overall, the performance of HARP-I was similar to SP-HR and SinMod at any wavelength.

In terms of nRMSE and DE, the three methods showed a clear trend across all the wavelengths: the error increased with the displacement level (see Figure 5). However, no major differences were found in any case. The average nRMSE and DE obtained using SP-HR, SinMod, and HARP-I (across all wavelengths and displacement levels) were $6.1 \pm 2.5 \%$ and $1.8 \pm 0.6^{\circ}$, $6.9 \pm 2.6 \%$ and $2.2 \pm 0.6^{\circ}$, and $5.6 \pm 2.6 \%$ and $1.8 \pm 0.6^{\circ}$ respectively, i.e., SP-HR and HARP-I showed slightly better performance than SinMod.

When looking at the variability in the error metrics, the behavior was similar to what was described before. The average standard deviations were $1.0 \pm 0.7 \%$ and $0.4 \pm 0.2^{\circ}$ for SP-HR, $1.1 \pm 0.6 \%$ and $0.5 \pm 0.2^{\circ}$ for SinMod, and $1.0 \pm 0.6 \%$ and $0.4 \pm 0.2^{\circ}$ for HARP-I, which means that almost no differences were found in terms of variability.

For both strain components, the error metrics behaved similarly to the errors in the displacement field, exhibiting a clear increasing trend with the motion levels (see third and fourth rows of Figure 5). However, HARP-I did not follow this trend for $\mathrm{WL}_{3}$ and SP-HR achieved bigger errors in the last displacement level of the $\mathrm{WL}_{7}$. Despite the bigger errors of HARP-I at the smallest displacement for $\mathrm{WL}_{3}$, SinMod and HARP-I always achieved better performances in both strain components for $\mathrm{WL}_{3}$ and $\mathrm{WL}_{5}$. For $\mathrm{WL}_{7}$, however, SP-HR performed better than SinMod but worse than HARP-I. In terms of averages (across wavelengths and displacement levels), for the circumferential strain the three methods reached errors similar to those found for displacements. However, for the radial component the error increased considerably when compared with displacement 


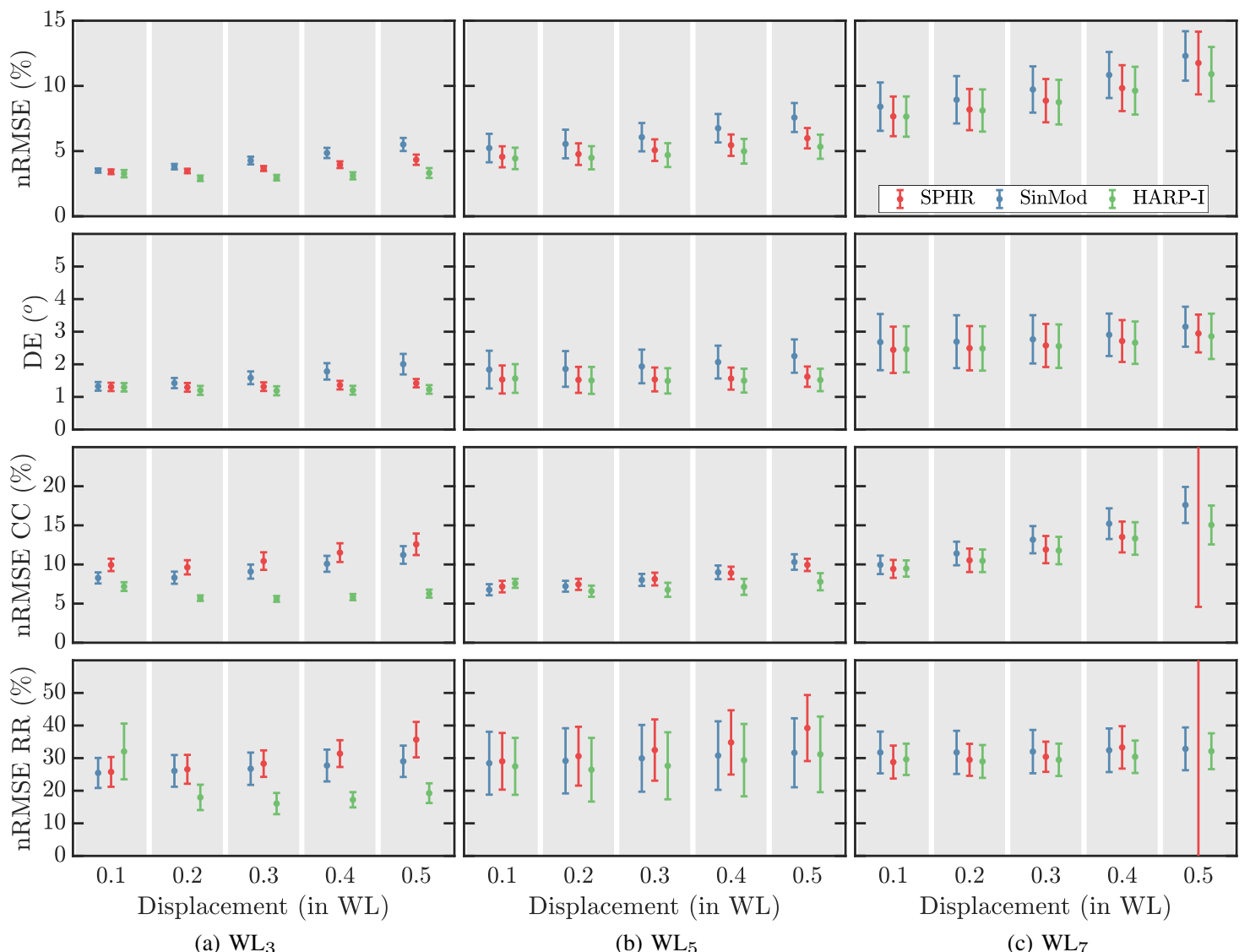

Fig. 5: Mean errors and deviations in the estimation of displacement magnitude (first row), direction (second row), circumferential strain (third row), and radial strain (fourth row) for wavelengths of (a) 2.9 ( $\mathrm{WL}_{3}$ ), (b) 4.9 (WL $\mathrm{WL}_{5}$ ), and (c) $6.9\left(\mathrm{WL}_{7}\right)$ pixels. The label in the $x$-axis denotes the amount of radial and angular displacement of the synthetic data (in the last case, the $x$-axis represents $r$ times the angular displacement, as stated by (19)), while in the $y$-axis, CC and RR stand for circumferential and radial strain components. For all the displacement levels and wavelengths, the performance of HARP-I was superior than SP-HR and SinMod.

magnitude and direction, and also with the circumferential part, reaching average values of $33.6 \pm 4.7$ for SP-HR, $29.7 \pm 2.9$ for SinMod, and $26.4 \pm 2.9$ for HARP-I.

In general, the three methods showed good performance estimating displacements and strains as the errors and differences between techniques were relatively small. This mean that SP-HR, SinMod, and HARP-I showed a similar behavior under ideal conditions.

\section{B. Noisy experiments}

Figure 6 (first and second rows) shows the error metrics for displacement magnitude and direction in the presence of noise. When compared with the noise-free results, the three methods performed worse (as expected) but keeping a similar difference in the error metrics between techniques, although HARP-I achieved the best results at all the displacement levels.

The average error across displacements, in terms of nRMSE and DE, were $8.0 \pm 2.0 \%$ and $2.7 \pm 0.7^{\circ}$ for SP-HR, $8.4 \pm 1.9 \%$ and $2.8 \pm 0.5^{\circ}$ for SinMod, and $7.1 \pm 2.1 \%$ and $2.4 \pm 0.6^{\circ}$ for HARP-I. When compared with the same results in the noise-free case, the errors were 1.3 and 1.5 times greater for SP-HR, 1.2 and 1.3 for SinMod, and 1.3 and 1.3 for HARP-I, which means that SinMod was slightly less sensitive to noise in the estimation of displacements. Although the increase in the average nRMSE and DE, the variability in error across the dataset did not experience a significant increase.

Figure 6 (third and fourth rows) shows the error metrics for the estimation of the strain components. The circumferential and radial components were better estimated with HARP-I, whereas SP-HR performed the worst. In the last case, similar to the noise-free case, bigger errors where induced in the estimation of strain at the last displacement level. The average errors for the circumferential and radial components were $25.8 \pm 14.9 \%$ and $41.5 \pm 10.4 \%$ for SP-HR, $21.8 \pm 10.8 \%$ and $34.2 \pm 5.0 \%$ for SinMod, and $15.4 \pm 4.5 \%$ and $30.7 \pm 7.1 \%$ for HARP-I. When compared with the noise-free experiment, errors obtained 


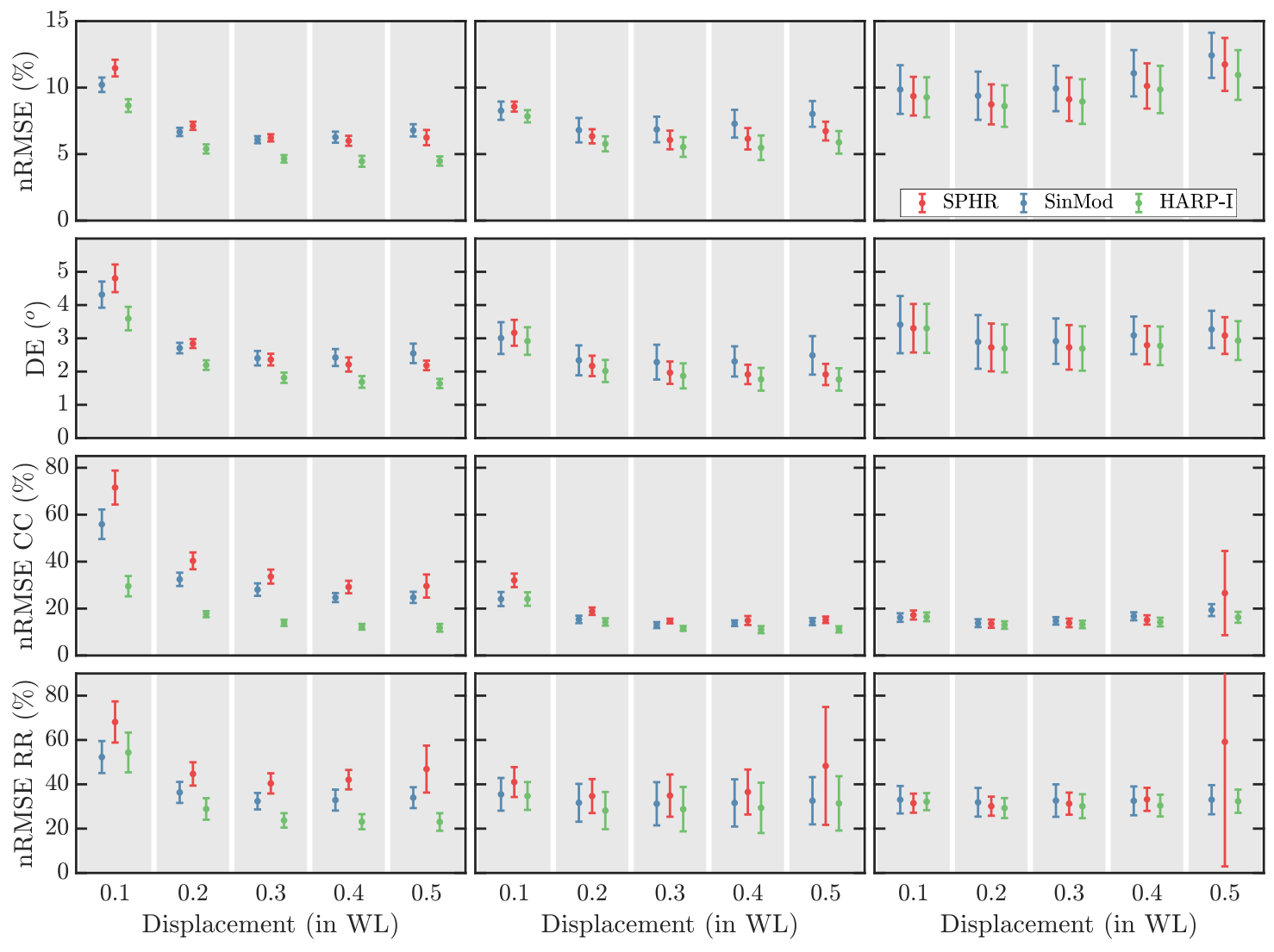

(a) $\mathrm{WL}_{3}$

(b) $\mathrm{WL}_{5}$

(c) $\mathrm{WL}_{7}$

Fig. 6: Mean errors and deviations in the estimation of displacement magnitude (first row), direction (second row), circumferential strain (third row), and radial strain (fourth row) for the noisy experiment for wavelengths of (a) 2.9 (WL $\mathrm{WL}_{3}$ ), (b) $4.9\left(\mathrm{WL}_{5}\right)$, and (c) $6.9\left(\mathrm{WL}_{7}\right)$ pixels. Strain estimations obtained SP-HR, SinMod, and HARP-I followed a similar behavior than the displacement magnitude and direction. However, the error increased due to the presence of noise.

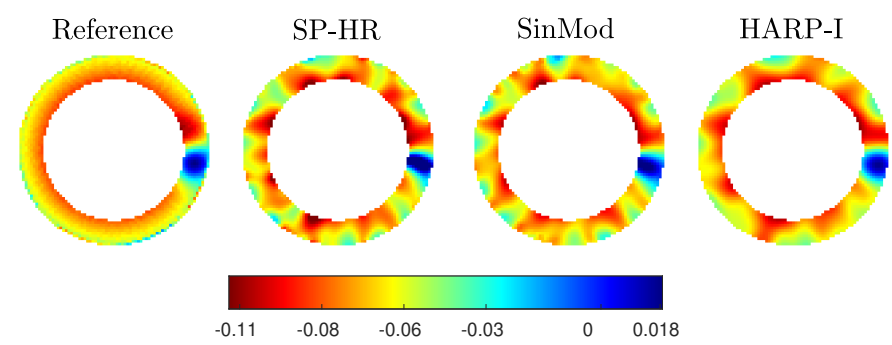

Fig. 7: Circumferential strain maps obtained with SP-HR, SinMod, and HARP-I for one representative sample of noisy data using $\mathrm{WL}_{5}$. Rapid spatial variations were observed in estimations made with SP-HR and SinMod, whereas more accurate and homogeneous results were obtained using HARP-I.

under noisy conditions were 2.3 and 1.2 times greater with SP-HR, 2.1 and 1.2 greater for SinMod, and 1.9 and 1.2 greater for HARP-I, indicating that derivatives estimated from SinMod and HARP-I results were less sensitive to noise than SP-HR.

Finally, Figures 7 and S1 (see Supporting Information) show the accuracy of HARP-I in the estimation of the position, shape, and strain values under noisy and noise-free conditions. Particularly, Figure S1 presents the results of an experiment conducted to determine the accuracy of HARP-I in the estimation of strain under different inclusion sizes.

\section{Motion estimation on healthy volunteers}

Figure 8 shows the left-ventricular strain maps of a subset of five healthy volunteers estimated using SP-HR, SinMod, and HARP-I, from tagged MR images. Although the strain maps at end-systole were similar between techniques in most cases, SP-HR gave results with severe artifacts, while SinMod and HARP-I showed smooth and similar strain maps. At late-diastole, severe artifacts were obtained with the three techniques, but to a lesser extent with HARP-I. In the same Figure, strain maps 

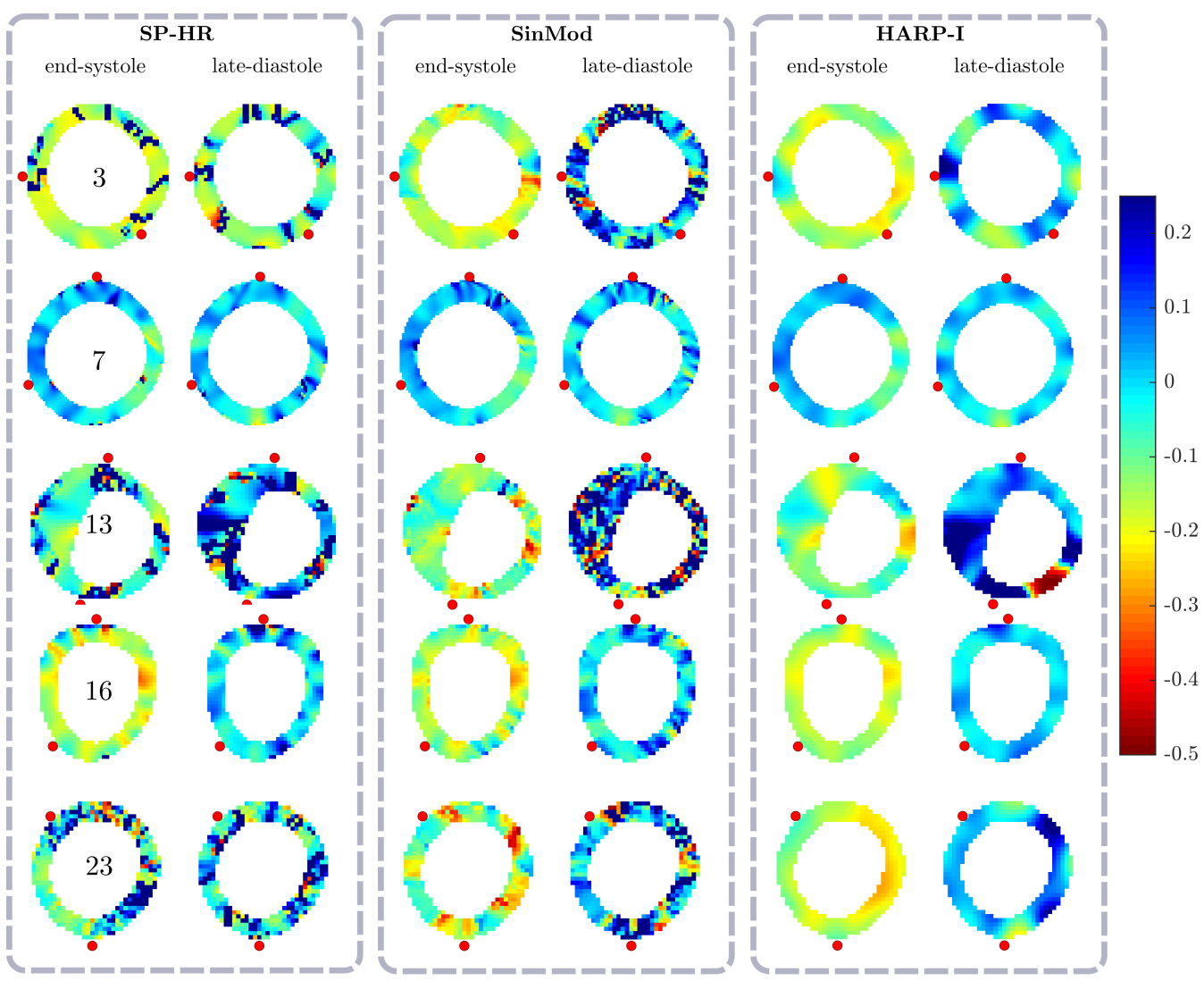

Fig. 8: Circumferential strain of a short-axis view of the left ventricle for 5 volunteers at end-systole and late-diastole (the last frame acquired). We used late-diastole to emphasize that in ECG triggered MR images, the cardiac cycle is not completely sampled. All the maps are presented in the reference cardiac phase (first frame) and were estimated using SP-HR (left), SinMod (middle), and HARP-I (right). The red dots indicate the insertion points of the RV walls into the LV. Each volunteer was identified with a number to make it easier to refer. The volunteers 7 and 13 presented abnormal strain patterns as they presented cardiomyopathies. The first case showed a hypokinetic LV and the second hypertrophic cardiomyopathy. SP-HR showed strain maps with artifacts on 3 of the 5 cases, whereas SinMod and HARP-I showed similar results at end-systole for most of the cases. Nonetheless, at late-diastole (where cardiac strain in healthy subjects should be small as the heart relaxes and come back to its reference position) some artifacts were observed with three methods in almost all cases. However, HARP-I showed a more stable behavior across the whole cardiac cycle.

of two patients, one with a hypokinetic LV (volunteer 7) and other with hypertrophic cardiomyopathy (volunteer 13), are presented.

To get an error measure of the motion and strain, we followed a similar approach as in [28], and for all the volunteers, we estimated the accumulated fraction of pixels with circumferential strains exceeding the unlikely high value of $50 \%$ (see Figure 9). For SP-HR and SinMod this metric increased to elevated levels, reaching maximum values 1.5 and 1.1 respectively. For HARP-I, on the other hand, the fraction remained always close to zero, with a maximum value of 0.02 .

In Figure 10 the temporal strain curves obtained with the three techniques are shown. With SP-HR and SinMod, highly unlikely strain values (bigger than 0.5) were not considered for the estimation of the curves, whereas with HARP-I all the values were used. Overall, the results given by the three methods were physiologically consistent and closer to clinical values, although SP-HR not always gave good results (see volunteers 11, 13 18, 21, 22, and 23 in Figure 10).

Finally, Figure 11 shows the estimated motion of the processed 3D data using 3D HARP-I and 3D SinMod. Each slice of the LV shows the circumferential strain and displacement field (arrows). HARP-I gave smoother strain maps than SinMod and displacements were closer to what we observed from the images. At basal level, the longitudinal displacement measured from the images was -7 and -11 pixels at the red and blue points (see Figures 11a and 11b), whereas with HARP-I was -7.2 and -10.8, and SinMod -7.6 and -13.1, which means that SinMod overestimated the longitudinal component of the displacement.

\section{DISCUSSION}

HARP-I was based on the same principles of the original HARP method but using a different approach to recover the tissue motion. Firstly, we obtained the harmonic phases by bandpass filtering the tagged MR images. Second, we corrected the phases 


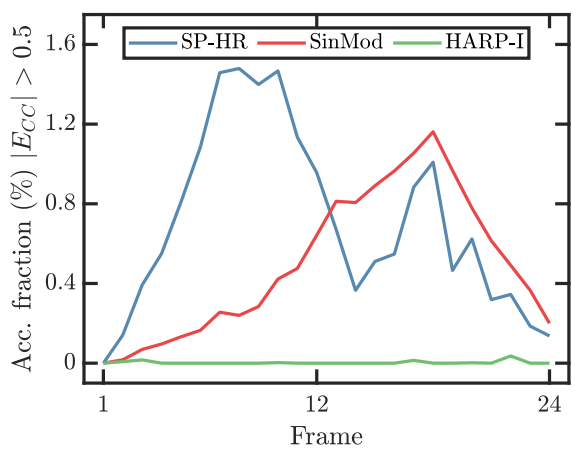

Fig. 9: Accumulated (across all volunteers) fraction of pixels with circumferential strain $\left(E_{C C}\right)$ with unlikely high values. The $\mathrm{X}$-axis denotes the frame of the cardiac cycle relative to the first one. The fraction was estimated as the number of pixels satisfying $\left|E_{C C}\right|>0.5$ divided by the number of pixels inside the left ventricle. Results were added together no matter if they shared the same number of frames (this explains why SP-HR and SinMod shows curves with increasing and decreasing behavior).
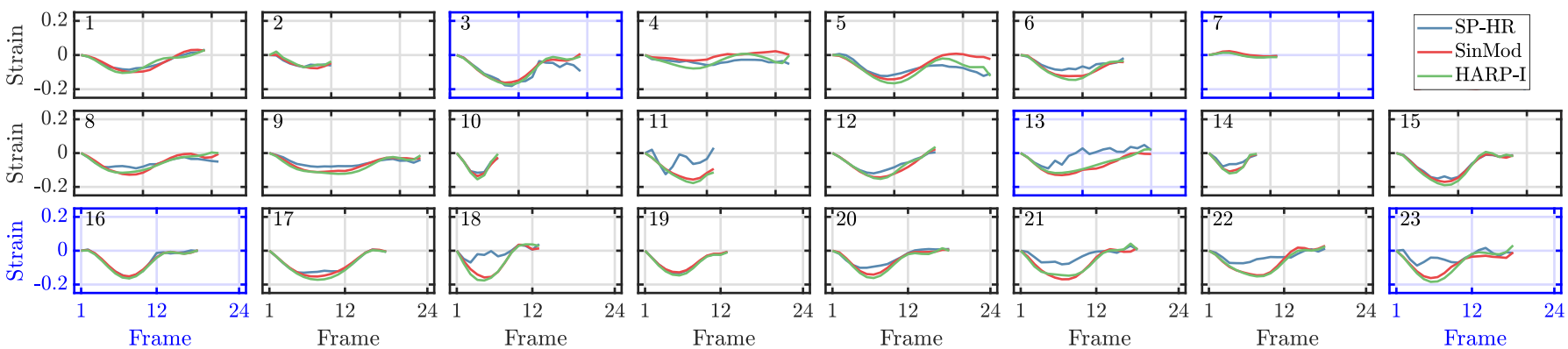

Fig. 10: Circumferential strain curves estimated with SP-HR, SinMod, and HARP-I for in-vivo data (highly unlikely strain values bigger than 0.5 were not considered for the estimation of the curve with SP-HR and SinMod methods, not so with HARP-I). The number on each plot is a label to make it easier to refer to the result of each volunteer. Results remarked with blue boxes are the same as those presented in Figure 8. The volunteers 7 and 13 presented abnormal strain patterns as they presented cardiomyopathies. The first case showed a hypokinetic LV and the second hypertrophic cardiomyopathy.

from wrapping artifacts, and then, we correct phase inconsistencies using distance matrices on the virtual coordinate system. Finally, similarly to HARP which treats the phase as a material property, we used the phase to recover, via RBF interpolations, the motion field at any time on a reference frame. The previous methodology gave us a new technique with similar capabilities to SP-HR and SinMod, but with improved results under ideal as well as under noisy conditions.

In the absence of noise, the proposed method worked similar to that of SP-HR and SinMod, reaching all the techniques the worst performance with $\mathrm{WL}_{7}$ (see Figure 5). For this wavelength, the over-smoothed image obtained by the small bandwidth of the filter did not allow the accurate tracking of the tissue, and sharp details as the stiffer inclusion were lost. Exactly the opposite happened with smaller wavelengths (i.e., bigger filter bandwidths), where more detailed and accurate motion and strain maps were obtained (see Figure 5 and 7).

One of the most relevant biomarkers of the left-ventricular function is the circumferential strain [12], [54], which can be directly calculated from displacement fields. However, a good estimation of the tissue motion does not ensure a similar performance of the strain estimate. The last is summarized in Figure 6, where in presence of small motions and noise, SP-HR estimated fairly well the displacement field but missestimated the strain maps when compared with SinMod. Furthermore, the nRMSE in the circumferential and radial components obtained with SP-HR (under noise-free and noisy conditions) became more significant at the highest displacement level for $\mathrm{WL}_{7}$ (see Figures 5 and 6), which is attributable to high DC contamination. HARP-I, on the other hand, gave the best estimations of both displacements and strain even at small motion levels, which is advantageous in the study of diseases where small displacements and strains are relevant [55].

HARP-I also demonstrated to be a reliable tool for the estimation strain from in-vivo data (see Figures 8 and 10), where strains similar to SP-HR and SinMod were obtained but with improved quality. The last assertion is also true for patient data with abnormal contraction patterns. For instance, in Figure 8, the end-systolic strain maps of the volunteers 7 (hypokinetic LV) and 13 (hypertrophic cardiomyopathy) obtained with HARP-I were comparable to SP-HR and SinMod, and sharp details were conserved (see the anterolateral and inferolateral parts of volunteer 7, and the anteroseptum for volunteer 13). In the case of the hypokinetic LV, small details were kept even for small motion levels and deformations. However three methods failed at diastolic cardiac phases mainly due to DC contamination of the harmonic images. 


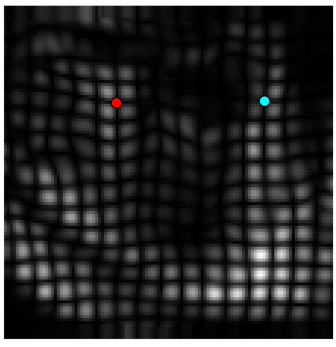

(a) Slice at frame 2

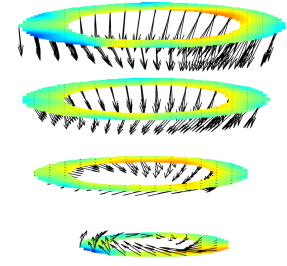

(c) HARP-I

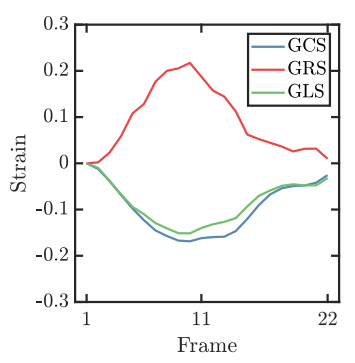

(e) HARP-I

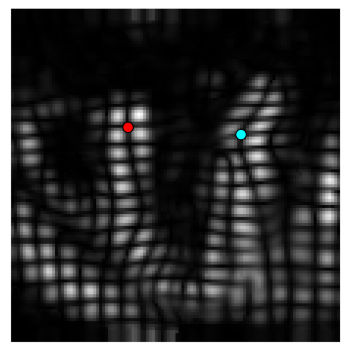

(b) Slice at frame 10

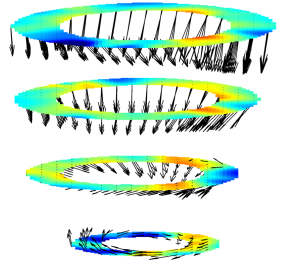

(d) SinMod

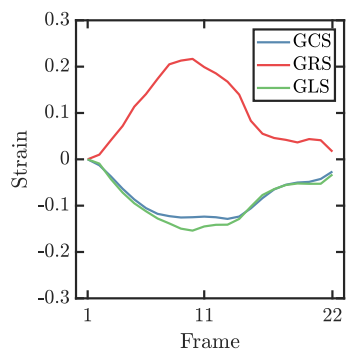

(f) SinMod

Fig. 11: Motion estimated from a 3D CSPAMM image of a healthy volunteer using HARP-I and SinMod. The figure shows a slice of the 3D volume at (a) frame 2 and (b) 10 of the cardiac cycle (the first frame was not used because of the lack of contrast between the cardiac walls and the blood pool) and the circumferential strain and displacement field (arrows) at frame 10 estimated using (c) HARP-I and (d) SinMod. The red and blue dot denotes the position of two points manually tracked. Figures (e) and (f) shows the global strains of the circumferential, radial, and longitudinal components (GCS, GRS, and GLS, respectively) obtained with HARP-I and SinMod. The strain maps were plotted in the range $[-0.5,0.2]$.

Looking carefully into the strain curves, we could observe that they are according to the diagnoses and MRI findings detailed in Table I. For subject 4, the Global Peak-Circumferential Strain (GPCS) was $-7.0 \%$, which is consistent with the decreased LV systolic function, and the dyskinetic motion explains the unusual shape. The hypokinetic LV of subject 7 explains the GPCS of $-1.4 \%$ and its shape. The patients with Duchenne muscular dystrophy (subjects 10,11, 12, and 14) presented a GPCS of $-15.3 \pm 2.6 \%$, which is slightly reduced compared to reference values [56]. Volunteer 13 exhibited the expected behavior in hypertrophic cardiomyopathy patients [57]. Additionally, we found a reduced GCPS of $-10.5 \%$ and $-11.6 \%$ in subjects 1 and 8 respectively, which had no known findings or diagnoses. Interestingly, for subject 2, the GCPS was $-7.1 \%$, which is surprisingly low for a person with an EF of 50\% (greater than 55\% is considered normal). The last could be explained by some pathology developed with almost preserved EF unknown by the authors. Excluding the data previously mentioned, the GCPS on healthy subjects was $-16.2 \pm 1.9 \%$ and almost no differences were found between SP-HR, SinMod, and HARP-I.

Until this point, it is crucial to notice that the quality of the harmonic images highly depends on the bandpass filter. Several tagging modalities (as those used to acquire the volunteer data) do not have a sinusoidal intensity, and therefore, the frequency domain contains higher harmonics and a DC component. [15]. Thus, the size of the filter should be adjusted to ensure the isolation of just one harmonic and avoid the low-frequency component. At low magnetic fields, however, regardless of the accurate fitting of the filter, obtaining a harmonic image is not always possible because the DC component recovers rapidly with $T 1$, which contaminates the harmonic peak early in the cardiac cycle. The last was observed in almost all the in-vivo experiments, where the image corruption at diastolic cardiac phases, did not allow the appropriate estimation of motion with any of the three methods (see Figure 8). These issues can be surpassed by using acquisition and reconstruction techniques such as CSPAMM [15] and MICSR [58] with the cost of increased scan time. 
An advantageous feature of HARP-I is that, although RBF interpolations removed rapid spatial variations from displacements and strain maps, there was no deterioration in the estimation of local characteristics of the tissue. In fact, under noise-free conditions and without DC component (as in CSPAMM or MICSR images), the shape and position of the stiffer inclusions of different sizes were correctly estimated in almost all the cases, failing just with the smallest (see Figure S2 in Supporting Information). Furthermore, in Figure 7 HARP-I correctly estimated the position, shape, and strain of the stiffer inclusion under noisy and DC-contaminated conditions, which could be determinant for the detection of infarcted tissue and other diseases.

Another feature of HARP-I which is shared with SP-HR and SinMod, is that none of them need a segmentation to estimate tissue motion, and similar motion-guided semi-automatic segmentation techniques as such introduced in [32] and [59] can also be implemented. However, the motion estimation with HARP-I depends on the availability of a unwrapped harmonic phase of high quality on the tissue of interest, which depends on the unwrapping method used. In this work, a Quality-guided Path Following algorithm was used, which performed well in all the cases as the path was constrained inside the geometry of the tissue, i.e., a segmentation was available. To avoid the segmentation without losing quality in the estimation of motion, unwrapping algorithms based on phase predictions with region growing [60] and graphcuts [61], [62] could be used.

When working with the 3D CSPAMM data, HARP-I worked better than SinMod in terms of strain smoothness and displacement field estimation. In fact, HARP-I was able to give accurate estimation displacement field in the longitudinal direction (the reference values were obtained manually from the images), whereas SinMod presented overestimated values. Additionally, as HARP-I estimates the motion between two frames directly (without intermediate estimations), less tracking artifacts were observed.

The efficiency of the proposed method, in terms of computation time, was also measured and compared against SP-HR and SinMod. In general, on a machine with an Intel Core i7-8700 CPU with 12 physical cores of $3.20 \mathrm{GHz}$ and $32 \mathrm{~Gb}$ of memory, HARP-I took around 6.0 seconds to process each data (phase-unwrapping and interpolation), while SP-HR took 1.4 seconds and SinMod 3.2. Additionally, for the 3D experiment, the execution times were 184 seconds with HARP-I (phaseunwrapping and interpolation) and 155 with SinMod (frame-by-frame estimation and Lagrangian tracking). In the last case, to avoid memory issues with HARP-I, we implemented a disjoint RBF interpolation scheme, in which the interpolator was defined on a equispaced downsampled set of points and evaluated incrementally in the reference frame. Overall, the three techniques shared similar processing times with the same order of magnitude, and no delays were introduced by HARP-I.

In this study, HARP-I was used to estimate the Lagrangian strain at a reference frame. However, with a small modification of the interpolator given in (7) Eulerian strain could also be obtained. If the interpolator is defined once at the first cardiac phase and evaluated at $\boldsymbol{X}_{V}\left(\boldsymbol{x}, t_{k}\right)$, the Eulerian displacement is obtained at frame $k$, which allows the estimation of the Eulerian strain tensor. This approach would be faster because it does not need the definition of multiple interpolators.

Additionally, we developed a new method to fix the temporal phase inconsistencies, which uses the same distance matrices needed for the RBF interpolation. With this method, we were able to correct all the phase jumps between frames without additional constraints on the tag-spacings and temporal resolution, making it robust to any combination of imaging parameters. On all the tested data, either in-silico and in-vivo, the correction worked in $100 \%$ of the cases. Therefore, even at the low temporal resolutions usually found in clinical and scientific setups (18 to 20 frames per cardiac cycle), HARP-I could work with even fewer frames without any bound on the total number. Although techniques like HARP Tracking Refinement Using Seeded Region Growing [35] and SP-HR [36] can also deal with large tissue motion between frames, in this work we found that SP-HR and SinMod failed in many cases due to high DC contamination.

Finally, when compared to further improvements of HARP, HARP-I addressed the same issues but with enhanced results. With HARP-I, smoothed results were obtained without loosing quality in the estimation and keeping small and sharp details. The amount of artifacts in the displacement field where highly reduced and they did not propagated to other cardiac phases. Although the used quality-guided unwrapping algorithm does not handle well corrupted phases, HARP-I demonstrated to have low sensitivity to DC contamination, maintaining its robustness even for highly deteriorated harmonic phases. The proposed RBF interpolation approach also dealt with mistracked boundary points and through-plane motion (which makes the tissue disappear) by interpolating or extrapolating the positions depending on the case. In the case of artifacts in the motion pattern, these were corrected using the polynomial fitting proposed in [48], which did not work with either SP-HR and SinMod.

\section{CONCLUSION}

We developed a new method for motion estimation from tagged MR images based on the same physical principle as HARP and SP-HR but with a different processing pipeline. HARP-I allows the estimation of motion in any direction with better results than SP-HR and SinMod techniques under highly demanding conditions as DC contamination, tag-lines fading, and image quality. HARP-I also showed to be more robust on estimating motion patterns with high temporal consistency, which was appreciated as artifacts-free strain patterns at almost any cardiac phase. Fast and robust extraction of motion gives HARP-I a potential in routine analysis of clinical exams to facilitate patient stratification. Secondly, the outcome of HARP-I could be used as high-quality observers while assimilating data into complex biomechanical models, e.g., to contribute to optimal therapeutical action [63]-[66]. 


\section{APPENDIX}

\section{A. Exact interpolation condition}

To determine the RBF weights associated with the interpolation function $g(\cdot)$ in (7), satisfying the exact interpolation condition $\boldsymbol{g}\left(\boldsymbol{X}_{V}\left(\boldsymbol{x}_{i}, t_{k}\right)\right)=\boldsymbol{x}_{i}$, the following linear system must be solved [44]:

$$
\Psi \lambda=G
$$

where $\lambda$ is a column vector of RBF weights, $G$ a column vector containing the deformed positions of each pixel (observations), and each element of the matrix $\boldsymbol{\Psi}$, for $m, n \in\left\{1, \cdots, N_{\text {pixels }}\right\}$, is given by:

$$
\Psi_{m n}=\psi\left(\left\|\boldsymbol{X}_{V}\left(\boldsymbol{x}_{m}, t_{k}\right)-\boldsymbol{X}_{V}\left(\boldsymbol{x}_{n}, t_{k}\right)\right\|\right) .
$$

\section{B. Evaluated norms in the RBF interpolator}

As the deformation map $\boldsymbol{\varphi}(\boldsymbol{X}, t)$ is invertible [43], there are two points $\boldsymbol{P} \in \boldsymbol{R}_{0}$ and $\boldsymbol{p} \in \boldsymbol{R}_{k}$ satisfying $\boldsymbol{X}\left(\boldsymbol{x}(\boldsymbol{p}), t_{k}\right)=$ $\boldsymbol{X}\left(\boldsymbol{P}, t_{0}\right)$ (see Figure 1). Therefore, the next relation holds:

$$
\boldsymbol{X}_{V}\left(\boldsymbol{P}, t_{0}\right)-\boldsymbol{X}_{V}\left(\boldsymbol{x}(\boldsymbol{p}), t_{k}\right)=\Delta \boldsymbol{n}_{\phi}
$$

where $\Delta \boldsymbol{n}_{\phi}(\boldsymbol{x})=\boldsymbol{n}_{\phi}\left(\boldsymbol{P}, t_{0}\right)-\boldsymbol{n}_{\phi}\left(\boldsymbol{p}, t_{k}\right)$ represents a noise difference.

Considering that the euclidean norm of the relation (24) relates to the noise difference, the evaluation of $\boldsymbol{X}_{V}\left(\boldsymbol{X}, t_{0}\right)$ in the interpolator in (7) gives an estimate of the target function on the reference domain.

\section{REFERENCES}

[1] M. J. Götte, T. Germans, I. K. Rüssel, J. J. Zwanenburg, J. T. Marcus, A. C. van Rossum, and D. J. van Veldhuisen, "Myocardial Strain and Torsion Quantified by Cardiovascular Magnetic Resonance Tissue Tagging. Studies in Normal and Impaired Left Ventricular Function," Journal of the American College of Cardiology, vol. 48, no. 10, pp. 2002-2011, nov 2006.

[2] A. Schuster, M. Paul, N. Bettencourt, G. Morton, A. Chiribiri, M. Ishida, S. Hussain, R. Jogiya, S. Kutty, B. Bigalke, D. Perera, and E. Nagel, "Cardiovascular magnetic resonance myocardial feature tracking for quantitative viability assessment in ischemic cardiomyopathy," International Journal of Cardiology, vol. 166, no. 2, pp. 413-420, jun 2013.

[3] K. Mangion, D. Carrick, J. Carberry, A. Mahrous, C. McComb, K. G. Oldroyd, H. Eteiba, M. Lindsay, M. McEntegart, S. Hood, M. C. Petrie, S. Watkins, A. Davie, X. Zhong, F. H. Epstein, C. E. Haig, and C. Berry, "Circumferential Strain Predicts Major Adverse Cardiovascular Events Following an Acute ST-Segment-Elevation Myocardial Infarction,” Radiology, vol. 290, no. 2, pp. 329-337, feb 2019.

[4] M. Cikes and S. D. Solomon, "Beyond ejection fraction: An integrative approach for assessment of cardiac structure and function in heart failure," European Heart Journal, vol. 37, no. 21, pp. 1642-1650, jun 2016.

[5] B. E. U. Burkhardt, M. N. Velasco Forte, S. Durairaj, I. Rafiq, I. Valverde, A. Tandon, J. Simpson, and T. Hussain, "Timely Pulmonary Valve Replacement May Allow Preservation of Left Ventricular Circumferential Strain in Patients with Tetralogy of Fallot," Frontiers in Pediatrics, vol. 5, p. 39, feb 2017.

[6] A. Schuster, G. Morton, S. T. Hussain, R. Jogiya, S. Kutty, K. N. Asrress, M. R. Makowski, B. Bigalke, D. Perera, P. Beerbaum, and E. Nagel, "The intra-observer reproducibility of cardiovascular magnetic resonance myocardial feature tracking strain assessment is independent of field strength," European Journal of Radiology, vol. 82, no. 2, pp. 296-301, 2013.

[7] T. Chitiboi and L. Axel, "Magnetic resonance imaging of myocardial strain: A review of current approaches," Journal of Magnetic Resonance Imaging, vol. 46, no. 5, pp. 1263-1280, 2017.

[8] M. S. Amzulescu, M. De Craene, H. Langet, A. Pasquet, D. Vancraeynest, A. C. Pouleur, J. L. Vanoverschelde, and B. L. Gerber, "Myocardial strain imaging: review of general principles, validation, and sources of discrepancies," European Heart Journal - Cardiovascular Imaging, vol. 20, no. 6, pp. 605-619, jun 2019.

[9] L. Chen, "Quantification of Left Ventricular Function with Contrast-Enhanced Harmonic Colour Doppler and a Semiautomated Boundary Detection Algorithm in Technically Difficult Patients: Feasibility, Accuracy, and Inter-observer Variability," European Journal of Echocardiography, vol. 2, no. 4, pp. 253-261, dec 2001.

[10] I. S. Salgo, W. Tsang, W. Ackerman, H. Ahmad, S. Chandra, M. Cardinale, and R. M. Lang, "Geometric assessment of regional left ventricular remodeling by three-dimensional echocardiographic shape analysis correlates with left ventricular function," Journal of the American Society of Echocardiography, vol. 25, no. 1, pp. 80-88, jan 2012.

[11] Y. Nagata, Y. Kado, T. Onoue, K. Otani, A. Nakazono, Y. Otsuji, and M. Takeuchi, "Impact of image quality on reliability of the measurements of left ventricular systolic function and global longitudinal strain in 2D echocardiography," Echo Research and Practice, vol. 5, no. 1, pp. 27-39, mar 2018.

[12] A. Young, "Assessment of cardiac performance with magnetic resonance imaging," Current Cardiology Reviews, vol. 2, no. 4, pp. 271-282, 2006.

[13] E. A. Zerhouni, D. M. Parish, W. J. Rogers, A. Yang, and E. P. Shapiro, "Human heart: Tagging with MR imaging - A new method for noninvasive assessment of myocardial motion," Radiology, vol. 169, no. 1, pp. 59-63, oct 1988.

[14] L. Axel and L. Dougherty, "MR imaging of motion with spatial modulation of magnetization." Radiology, vol. 171, no. 3, pp. 841-845, 1989.

[15] S. E. Fischer, G. C. McKinnon, S. E. Maier, and P. Boesiger, "Improved myocardial tagging contrast," Magnetic Resonance in Medicine, vol. 30, no. 2, pp. 191-200, aug 1993.

[16] S. E. Fischer, G. C. McKinnon, M. B. Scheidegger, W. Prins, D. Meier, and P. Boesiger, "True myocardial motion tracking," Magnetic Resonance in Medicine, vol. 31, no. 4, pp. 401-413, apr 1994.

[17] L. Axel, A. Montillo, and D. Kim, "Tagged magnetic resonance imaging of the heart: A survey," Medical Image Analysis, vol. 9, no. 4 SPEC. ISS., pp. 376-393, 2005.

[18] S. Ryf, M. A. Spiegel, M. Gerber, and P. Boesiger, "Myocardial tagging with 3D-CSPAMM," Journal of Magnetic Resonance Imaging, vol. 16, no. 3, pp. 320-325, sep 2002.

[19] A. K. Rutz, S. Ryf, S. Plein, P. Boesiger, and S. Kozerke, "Accelerated whole-heart 3D CSPAMM for myocardial motion quantification," Magnetic Resonance in Medicine, vol. 59, no. 4, pp. 755-763, apr 2008.

[20] M. A. Guttman, J. L. Prince, and E. R. McVeigh, "Tag and Contour Detection in Tagged MR Images of the Left Ventricle," IEEE Transactions on Medical Imaging, vol. 13, no. 1, pp. 74-88, 1994

[21] A. A. Young, "Model tags: Direct three-dimensional tracking of heart wall motion from tagged magnetic resonance images," Medical Image Analysis, vol. 3, no. 4, pp. 361-372, dec 1999. 
[22] L. Dougherty, J. C. Asmuth, A. S. Blom, L. Axel, and R. Kumar, "Validation of an optical flow method for tag displacement estimation," IEEE Transactions on Medical Imaging, vol. 18, no. 4, pp. 359-363, 1999.

[23] R. Chandrashekara, R. Mohiaddin, R. Razavi, and D. Rueckert, "Nonrigid image registration with subdivision lattices: Application to cardiac mr image analysis," in Proceedings of the 10th International Conference on Medical Image Computing and Computer-Assisted Intervention - Volume Part I, ser MICCAI'07. Berlin, Heidelberg: Springer-Verlag, 2007, p. 335-342.

[24] M. Genet, C. T. Stoeck, C. von Deuster, L. C. Lee, and S. Kozerke, "Equilibrated warping: Finite element image registration with finite strain equilibrium gap regularization," Medical Image Analysis, vol. 50, pp. 1-22, dec 2018.

[25] K. Škardová, M. Rambausek, R. Chabiniok, and M. Genet, "Mechanical and Imaging Models-Based Image Registration," in Lecture Notes in Computational Vision and Biomechanics. Springer Netherlands, oct 2019, vol. 34, pp. 77-85.

[26] N. F. Osman, W. S. Kerwin, E. R. McVeigh, and J. L. Prince, "Cardiac motion tracking using CINE harmonic phase (HARP) magnetic resonance imaging," Magnetic Resonance in Medicine, vol. 42, no. 6, pp. 1048-1060, dec 1999.

[27] N. F. Osman, E. R. McVeigh, and J. L. Prince, "Imaging heart motion using harmonic phase MRI," IEEE Transactions on Medical Imaging, vol. 19, no. 3, pp. 186-202, 2000

[28] T. Arts, F. W. Prinzen, T. Delhaas, J. R. Milles, A. C. Rossi, and P. Clarysse, "Mapping displacement and deformation of the heart with local sine-wave modeling," IEEE Transactions on Medical Imaging, vol. 29, no. 5, pp. 1114-1123, may 2010.

[29] Z. Qian, D. N. Metaxas, and L. Axel, "Extraction and tracking of MRI tagging sheets using a 3D gabor filter bank," Annual International Conference of the IEEE Engineering in Medicine and Biology - Proceedings, pp. 711-714, 2006.

[30] T. Chen and L. Axel, "Using gabor filter banks and temporal-spatial constraints to compute 3D myocardium strain," Annual International Conference of the IEEE Engineering in Medicine and Biology - Proceedings, pp. 4755-4758, 2006.

[31] H. Wang and A. A. Amini, "Cardiac motion and deformation recovery from MRI: A review," IEEE Transactions on Medical Imaging, vol. 31, no. 2, pp. 487-503, 2012 .

[32] A. M. Khalifa, A. B. M. Youssef, and N. F. Osman, "Improved Harmonic Phase (HARP) method for motion tracking a tagged cardiac MR images," Annual International Conference of the IEEE Engineering in Medicine and Biology - Proceedings, vol. 7 VOLS, pp. 4298-4301, 2005.

[33] S. R. Tecelão, J. J. Zwanenburg, J. P. Kuijer, and J. T. Marcus, "Extended harmonic phase tracking of myocardial motion: Improved coverage of myocardium and its effect on strain results," Journal of Magnetic Resonance Imaging, vol. 23, no. 5, pp. 682-690, 2006.

[34] L. Florack, H. Van Assen, and A. Suinesiaputra, "Dense multiscale motion extraction from cardiac cine MR tagging using harp technology," Proceedings of the IEEE International Conference on Computer Vision, 2007.

[35] X. Liu, E. Murano, M. Stone, and J. L. Prince, "HARP tracking refinement using seeded region growing," 2007 4th IEEE International Symposium on Biomedical Imaging: From Nano to Macro - Proceedings, pp. 372-375, 2007.

[36] X. Liu and J. L. Prince, "Shortest path refinement for motion estimation from tagged MR images," IEEE Transactions on Medical Imaging, vol. 29, no. 8 , pp. 1560-1572, 2010.

[37] S. M. ElDeeb and A. S. Fahmy, "Accurate harmonic phase tracking of tagged MRI using locally-uniform myocardium displacement constraint," Medical Engineering and Physics, vol. 38, no. 11, pp. 1305-1313, 2016.

[38] H. Liu, J. Wang, X. Xu, E. Song, Q. Wang, R. Jin, C. C. Hung, and B. Fei, "A robust and accurate center-frequency estimation (RACE) algorithm for improving motion estimation performance of SinMod on tagged cardiac MR images without known tagging parameters," Magnetic Resonance Imaging, vol. 32, no. 9, pp. 1139-1155, 2014.

[39] H. Wang and A. A. Amini, "Cardiac motion tracking with multilevel B-splines and SinMod from tagged MRI," J. B. Weaver and R. C. Molthen, Eds., vol. 7965. International Society for Optics and Photonics, mar 2011, p. 796520.

[40] K. M. Moerman, A. M. Sprengers, C. K. Simms, R. M. Lamerichs, J. Stoker, and A. J. Nederveen, "Validation of SPAMM tagged MRI based measurement of 3D soft tissue deformation," Medical Physics, vol. 38, no. 3, pp. 1248-1260, 2011.

[41] H. Wang and A. a. Amini, "Cardiac deformation analysis using 3D SinMod from 3D CSPAMM tagged MRI," in Proceedings Volume 8672, Medical Imaging 2013: Biomedical Applications in Molecular, Structural, and Functional Imaging, vol. 8672, 2013, p. 86720B.

[42] E. S. H. Ibrahim, J. Stojanovska, A. Hassanein, C. Duvernoy, P. Croisille, R. Pop-Busui, and S. D. Swanson, "Regional cardiac function analysis from tagged MRI images. Comparison of techniques: Harmonic-Phase (HARP) versus Sinusoidal-Modeling (SinMod) analysis," Magnetic Resonance Imaging, vol. 54, no. April, pp. 271-282, 2018.

[43] M. E. Gurtin, E. Fried, and L. Anand, The Mechanics and Thermodynamics of Continua. Cambridge: Cambridge University Press, 2010.

[44] M. D. Buhmann, Radial Basis Functions: Theory and Implementations. Cambridge: Cambridge University Press, 2003.

[45] T. Poggio and F. Girosi, "Networks for Approximation arid Learning," Proceedings of the IEEE, vol. 78, no. 9, pp. 1481-1497, 1990.

[46] D. C. Ghiglia and M. D. Pritt, Two-dimensional phase unwrapping: theory, algorithms, and software. Wiley, 1998.

[47] A. D. Gilliam and F. H. Epstein, "Automated motion estimation for 2-D cine DENSE MRI," IEEE Transactions on Medical Imaging, vol. 31, no. 9, pp. 1669-1681, 2012

[48] B. S. Spottiswoode, X. Zhong, A. T. Hess, C. M. Kramer, E. M. Meintjes, B. M. Mayosi, and F. H. Epstein, "Tracking myocardial motion from cine DENSE images using spatiotemporal phase unwrapping and temporal fitting," IEEE Transactions on Medical Imaging, vol. 26, no. 1, pp. 15-30, 2007.

[49] J. C. Carr, R. K. Beatson, B. C. McCallum, W. R. Fright, T. J. McLennan, and T. J. Mitchell, "Smooth surface reconstruction from noisy range data," in Proceedings of the 1st International Conference on Computer Graphics and Interactive Techniques in Australasia and South East Asia, GRAPHITE '03. New York, New York, USA: ACM Press, 2003, p. 119.

[50] X. Zhong, B. S. Spottiswoode, C. H. Meyer, C. M. Kramer, and F. H. Epstein, "Imaging three-dimensional myocardial mechanics using navigator-gated volumetric spiral cine DENSE MRI," Magnetic Resonance in Medicine, vol. 64, no. 4, pp. 1089-1097, jun 2010.

[51] G. J. Wehner, L. Jing, C. M. Haggerty, J. D. Suever, J. Chen, S. M. Hamlet, J. A. Feindt, W. Dimitri Mojsejenko, M. A. Fogel, and B. K. Fornwalt, "Comparison of left ventricular strains and torsion derived from feature tracking and DENSE CMR," Journal of Cardiovascular Magnetic Resonance, vol. 20, no. 1, p. 63, dec 2018 .

[52] E.-S. H. Ibrahim, "Myocardial tagging by Cardiovascular Magnetic Resonance: evolution of techniques-pulse sequences, analysis algorithms, and applications," Journal of Cardiovascular Magnetic Resonance, vol. 13, no. 1, p. 36, jul 2011.

[53] J. Mura, A. M. Pino, J. Sotelo, I. Valverde, C. Tejos, M. E. Andia, P. Irarrazaval, and S. Uribe, "Enhancing the Velocity Data From 4D Flow MR Images by Reducing its Divergence," IEEE Transactions on Medical Imaging, vol. 35, no. 10, pp. 2353-2364, oct 2016.

[54] C. Petitjean, N. Rougon, and P. Cluzel, "Assessment of myocardial function: A review of quantification methods and results using tagged MRI," Journal of Cardiovascular Magnetic Resonance, vol. 7, no. 2, pp. 501-516, 2005.

[55] D. A. Auger, K. C. Bilchick, J. A. Gonzalez, S. X. Cui, J. W. Holmes, C. M. Kramer, M. Salerno, and F. H. Epstein, "Imaging left-ventricular mechanical activation in heart failure patients using cine DENSE MRI: Validation and implications for cardiac resynchronization therapy," Journal of Magnetic Resonance Imaging, vol. 46, no. 3, pp. 887-896, sep 2017.

[56] K. N. Hor, J. Wansapura, L. W. Markham, W. Mazur, L. H. Cripe, R. Fleck, D. W. Benson, and W. M. Gottliebson, "Circumferential Strain Analysis Identifies Strata of Cardiomyopathy in Duchenne Muscular Dystrophy. A Cardiac Magnetic Resonance Tagging Study," Journal of the American College of Cardiology, vol. 53, no. 14, pp. 1204-1210, 2009.

[57] M. Y. Jeung, P. Germain, P. Croisille, S. E. Ghannudi, C. Roy, and A. Gangi, "Myocardial tagging with MR imaging: Overview of normal and pathologic findings," Radiographics, vol. 32, no. 5, pp. 1381-1398, 2012.

[58] M. NessAiver and J. L. Prince, "Magnitude image CSPAMM reconstruction (MICSR)," Magnetic Resonance in Medicine, vol. 50, no. 2, pp. 331-342, 2003. 
[59] C. A. Miller, A. Borg, D. Clark, C. D. Steadman, G. P. McCann, P. Clarysse, P. Croisille, and M. Schmitt, "Comparison of local sine wave modeling with harmonic phase analysis for the assessment of myocardial strain," Journal of Magnetic Resonance Imaging, vol. 38, no. 2, pp. 320-328, 2013.

[60] D. A. Auger, X. Cai, C. Sun, and F. H. Epstein, "Improved phase unwrapping algorithm for automatic cine DENSE strain analysis using phase predictions and region growing," in Proceedings of the 27th Annual Meeting of the ISMRM. ISMRM, 2018.

[61] J. M. Bioucas-Dias and G. Valadão, "Phase unwrapping via graph cuts," IEEE Transactions on Image Processing, vol. 16, no. 3, pp. 698-709, 2007.

[62] B. A. Venkatesh, H. Gupta, S. G. Lloyd, L. Dell 'Italia, and T. S. Denney, "3D left ventricular strain from unwrapped harmonic phase measurements," Journal of Magnetic Resonance Imaging, vol. 31, no. 4, pp. 854-862, apr 2010.

[63] A. Imperiale, R. Chabiniok, P. Moireau, and D. Chapelle, "Constitutive parameter estimation methodology using tagged-MRI data," in Lecture Notes in Computer Science (including subseries Lecture Notes in Artificial Intelligence and Lecture Notes in Bioinformatics), vol. 6666 LNCS. Springer, Berlin, Heidelberg, 2011, pp. 409-417.

[64] R. Chabiniok, P. Moireau, P. F. Lesault, A. Rahmouni, J. F. Deux, and D. Chapelle, "Estimation of tissue contractility from cardiac cine-MRI using a biomechanical heart model," Biomechanics and Modeling in Mechanobiology, vol. 11, no. 5, pp. 609-630, may 2012.

[65] C. Bertoglio, P. Moireau, and J.-F. Gerbeau, "Sequential parameter estimation for fluid-structure problems: Application to hemodynamics," International Journal for Numerical Methods in Biomedical Engineering, vol. 28, no. 4, pp. 434-455, apr 2012.

[66] M. Hadjicharalambous, L. Asner, R. Chabiniok, E. Sammut, J. Wong, D. Peressutti, E. Kerfoot, A. King, J. Lee, R. Razavi, N. Smith, G. Carr-White, and D. Nordsletten, "Non-invasive Model-Based Assessment of Passive Left-Ventricular Myocardial Stiffness in Healthy Subjects and in Patients with Non-ischemic Dilated Cardiomyopathy," Annals of Biomedical Engineering, vol. 45, no. 3, pp. 605-618, mar 2017. 